ARTículO DE REVISIÓN

\title{
Neuropatía diabética
}

Hermelinda C. Pedrosa ${ }^{1 *}$, José Daniel Braver ${ }^{2}$, Luiz Clemente Rolim ${ }^{3}$, Helena Schmid ${ }^{3}$, María Regina Calsolari ${ }^{3}$, Graciela Fuente ${ }^{4}$ y Ariel Odriozola ${ }^{5}$

${ }^{1}$ Coordinadora, Brasil; ${ }^{2}$ Coordinador, Argentina; ${ }^{3}$ Integrante, Brasil; ${ }^{4}$ Integrante, Argentina; ${ }^{5}$ Autor invitado, España. Grupo Neuropatía Autonómica, Asociación Latinoamericana de Diabetes

\section{RESUMEN}

La neuropatía es un diagnostico de exclusión, pudiendo no estar asociada a la diabetes. Se puede presentar en pacientes con diabetes y ser tratable con diferentes opciones. Existen un número de opciones terapéuticas para la neuropatía diabética sintomática, pudiendo haber un $50 \%$ de neuropatías en pacientes con diabetes totalmente asintomáticas. Por eso, es importante realizar evaluaciones apropiadas para descartar la presencia de la neuropatía. Reconocer y tratar la neuropatía autonómica puede aliviar los síntomas, reducir secuelas y mejorar la calidad de vida. Optimizar el control de la diabetes inclusive la glicemia y los factores del riesgo como la hipertensión arterial, dislipidemia y peso, necesita ser alcanzado lo más precozmente para prevenir o retrasar la progresión de la neuropatía en pacientes con diabetes mellitus tipo 1 y tipo 2. El control de la glucosa se ha mostrado más impactante entre los pacientes con diabetes tipo 1. Mientras que

\section{ABSTRACT}

Neuropathy is a diagnosis of exclusion as it may be caused by other diseases and nondiabetic neuropathies may be present among people with diabetes. It also may be treatable by diferent therapeutic and specific approaches for those who have symptomatic diabetic neuropathy meanwhile $50 \%$ of neuropathies are totally asymptomatic. Therefore, appropriate evaluation should be performed to confirm or rule out the presence of neuropathy. Diabetic autonomic neuropathy needs to be recognized and treated in order to relieve symptoms, reduce sequelae and improve quality of life. Diabetes control including glycemia and risk factors such as hypertension, dyslipidemia and weight control need to be achieved as early as possible to prevent and delay its progression in both types of diabetes. Glycemia control has proved to have more impact among type 1 diabetes while in type 2 diabetes patients a multifactorial management has more bene- 
la abordaje en foma multifactorial se comprobó tener más beneficio, especialmente dirigida a la prevención de la neuropatía autonómica cardiovascular, en pacientes con diabetes mellitus tipo 2 .

Palabras clave: Neuropatía diabética. Diabetes. Neuropatía autonómica. Neuropatía autonómica cardiovascular. fit, mainly to prevent cardiovascular autonomic neuropathy. (Rev ALAD. 2019;9:72-91) Corresponding author: Hermelinda C. Pedrosa, pedrosa.hc@diabetes.org.br, pedrosa.hc@globo.com

Key words: Diabetic neuropathy. Diabetes. Autonomic neuropathy. Cardiovascular autonomic neuropathy.

\section{INTRODUCCIÓN}

La neuropatía diabética (ND) es la complicación crónica más prevalente de la diabetes mellitus (DM) y representa un grupo heterogéneo de condiciones que afectan diferentes partes del sistema nervioso con diversas manifestaciones clínicas. Por esta razón es fundamental excluir otras causas de neuropatías $^{1-3}$.

La actual clasificación de las ND considera tres grandes grupos: difusas, mononeuropatías y polirradiculopatías ${ }^{3}$. Las difusas incluyen la polineuropatía simétrica distal (PNSD), que es la más frecuente, y las neuropatías autonómicas diabéticas (NAD). Es necesario considerar que las características anatómicas de las fibras del sistema nervioso autónomo (SNA) las hacen susceptibles a la noxa metabólica propia de la enfermedad de base, así como también de las subsiguientes vías relacionadas con la hiperglucemia. Por ello debe aceptarse que en forma simultánea con la PNSD el paciente puede presentar $\mathrm{NAD}^{2}$. La tabla 1 muestra la actual clasificación de las $\mathrm{ND}^{3}$.

Las NAD son desórdenes del SNA asociado a la diabetes y otras alteraciones metabólicas de la prediabetes después de excluir otras causas. Afectan al sistema nervioso parasimpático y al
TABLA 1. Clasificación actual de las ND

\begin{tabular}{|l}
\hline ND más frecuentes \\
\hline A. Neuropatía difusa \\
Polineuropatía distal simétrica \\
- Fibras delgadas \\
- Fibras gruesas \\
- Mixta \\
\hline Autonómicas \\
Cardiovascular \\
- Reduccion de la variabilidad FC \\
- Taquicardia de reposo \\
- HO \\
- Muerte súbita \\
Gastrointestinal \\
- Gastroparesia \\
- Diarreas/constipación \\
Urogenital \\
- Vejiga neurogénica \\
- ED \\
Disfunción sudomotora \\
- Anidrosis, hihidrosis \\
Hipoglucemia inadvertida \\
Disfunción pupilar
\end{tabular}

ND atípicas

B. Mononeuropatías (mononeuritis múltiple)

- Cranianas o del nervio periférico (III par craniano, nervios ulnar, mediano, femoral, peroneal)

- Mononeuritis múltiple

C. Radiculopatía o polirradiculopatía

- Polirradiculopatía lumbosacra, amiotrofia proximal motora

- Radiculopatía torácica

Neuropatías no diabéticas comunes en pacientes con diabetes

- Compresivas

- Polineuropatía inflamatoria desmielinizante crónica

- Neuropatía de plexo radicular

- Neuropatía dolorosa aguda de fibras finas (inducida por tratamiento)

ED: disfunción eréctil; FC: frecuencia cardíaca; HO: hipotensión ortostática; ND: neuropatías diabéticas.

Adaptada de Pop-Busui, et al. 2017³. 
simpático con una sintomatología que varía dependiendo del órgano afectado y que debe ser investigada mediante la historia clínica y el examen físico ${ }^{1-3}$. Dado que el SNA está compuesto por fibras amielínicas y de pequeño diámetro, su compromiso es precoz en la evolución de la enfermedad $^{2}$.

Globalmente se considera que las formas sintomáticas son raras. La neuropatía autonómica cardiovascular (NAC) es la más frecuente y la más estudiada, pero las manifestaciones clínicas gastrointestinales, genitourinarias y pseudomotoras, la hipoglucemia sin aviso y la disfunción pupilar necesitan ser consideradas en el tratamiento optimizado de pacientes con DM. Además, el diagnóstico y tratamiento de las NAD puede mejorar síntomas, lo que comporta una mejoría de la calidad de vida $(\mathrm{Q} O \mathrm{~L})^{3}$. Los principales signos y síntomas de las NAD aparecen en la tabla 2.

\section{RECOMENDACIONES CON BASE EN EL GRADO DE EVIDENCIA ${ }^{3}$}

- Síntomas y signos de neuropatía autonómica deben ser considerados en pacientes con complicaciones microvasculares y neuropáticas. E

- En la aparición de los síntomas y signos de la NAC deben ser realizadas otras pruebas a fin de excluirse otras comorbilidades o efectos colaterales de fármacos que podrían confundirse con manifestaciones de la NAC. E

- Se debe considerar siempre evaluar los síntomas y signos de NAC en los pacientes con hipoglucemia inadvertida o asintomática. C

- Optimizar el control de la glucemia en forma temprana permite la prevención y retardo del desarrollo de la NAC en pacientes con diabetes tipo 1. A

- Un tratamiento multifactorial que abarque el control de la glucemia y los factores de riesgo cardiovasculares previene la aparición de la NAC en la DM tipo 2 (DM2). C

\section{MANIFESTACIONES CLÍNICAS DE LA NEUROPATÍA AUTONÓMICA CARDIOVASCULAR}

La NAC está asociada a la taquicardia en reposo, la intolerancia al ejercicio, la hipotensión ortostática (HO), el síncope, la inestabilidad cardiovascular intraoperatoria, y el infarto de miocardio y la isquemia silentes ${ }^{1-3}$. El aumento de la mortalidad y la aparición de cualquier evento cardiovascular y disfunción miocárdica fueron verificadas en un metaanálisis de 15 estudios $^{4}$, que en total incluían a 2,900 pacientes con diabetes. El estudio evidenció un riesgo aumentado de mortalidad cardiovascular en aquellos que tenían NAC, con un riesgo relativo (RR) de 3.45 (IC 95\%: 2.66-4.47), lo que también se ha observado en otros estudios poblacionales ${ }^{5-9}$ que incluían pacientes con DM tipo 1 (DM1) y DM2. En el estudio DIAD ${ }^{8}$, que incluyó 1,123 pacientes con diagnóstico de $\mathrm{DM} 2$, se evaluó la presencia de isquemia miocárdica asintomática y de NAC; se observó que la NAC fue un importante predictor de isquemia silente y eventos cardiovasculares posteriores.

La prevalencia de NAC varía considerablemente y reflejan los diferentes test y criterios diagnósticos utilizados en la población estudiada. La NAC afecta a pacientes de todas las edades, con HbA1c (hemoglobina glicada) elevada, jóvenes y especialmente mujeres, afectando al 20\% de jóvenes con DM1 o 
TABLA 2. Manifestaciones clínicas de las neuropatías autonómicas diabéticas

\begin{tabular}{|c|c|c|c|}
\hline NAC & Gastrointestinal & Urogenital & Sudomotor \\
\hline $\begin{array}{l}\text { Taquicardia de reposo } \\
\text { - Regulación anormal de la } \\
\text { presión arterial } \\
\text { - Dipper/Non dipper }\end{array}$ & $\begin{array}{l}\text { Gastroparesia } \\
\text { - Náuseas } \\
\text { - Pérdida apetito } \\
\text { - Saciedad temprana } \\
\text { - Vomito posprandial } \\
\text { - Diabetes notable }\end{array}$ & $\begin{array}{l}\text { Disfunción vesical } \\
\text { - Frecuencia } \\
\text { - Urgencia } \\
\text { - Nicturia } \\
\text { - Retención } \\
\text { - Incontinencia } \\
\text { - Vacilación } \\
\text { - Jato débil }\end{array}$ & $\begin{array}{l}\text { Piel seca } \\
\text { - Anhidrosis } \\
\text { - Sudor profuso (cabeza y tórax) }\end{array}$ \\
\hline $\begin{array}{l}\text { Hipotensión ortostática y } \\
\text { taquicardia o bradicardia } \\
\text { ortostáticas e incompetencia } \\
\text { cronotrópica } \\
\text { - Mareo } \\
\text { - Debilidad } \\
\text { - Desmayo } \\
\text { - Afectación visual } \\
\text { - Síncope }\end{array}$ & $\begin{array}{l}\text { Disfunción esofágica } \\
\text { - Acidez } \\
\text { - Disfagia a sólidos }\end{array}$ & $\begin{array}{l}\text { Disfunción sexual masculina } \\
\text { - Disfunción eréctil } \\
\text { - Disminución de la libido } \\
\text { - Eyaculación anormal }\end{array}$ & \\
\hline Intolerancia al ejercicio & $\begin{array}{l}\text { Diarrea diabética } \\
\text { - Profusa y acuosa } \\
\text { - Incontinencia fecal } \\
\text { - Puede alternar con } \\
\text { constipación }\end{array}$ & $\begin{array}{l}\text { Disfunción sexual femenina } \\
\text { - Disminución del deseo sexual } \\
\text { - Dispareunia } \\
\text { - Alteración de la lubricación } \\
\text { - Disminución de la excitación } \\
\text { sexual }\end{array}$ & \\
\hline
\end{tabular}

NAC: neuropatía autonómica cardíaca.

Adaptada de Pop-Busui, et al. $2017^{3}$.

$\mathrm{DM} 2^{10}$. Además de esto puede ya estar presente en pacientes con intolerancia a la glucosa, resistencia a la insulina y síndrome metabólico ${ }^{11-13}$. En pacientes recientemente diagnosticados con DM1 la prevalencia de la NAC es más baja ${ }^{14}$, pero aumenta substancialmente con la duración de la DM. Los estudios DCCT/EDIC (Diabetes Control and Complications Trial/ Epidemiology of Diabetes Interventions and Complications) observaron tasas del 30\% después 20 años, pero el control intensivo de la glucemia redujo el riesgo de incidencia de NAC en el estudio DCCT en un $45 \%$ y en un $31 \%$ en el estudio EDIC ${ }^{15-17}$. En la DM2 la prevalencia de NAC también aumenta con la duración de la diabetes y afecta al 60\% después de 15 años $^{1,18}$.

En relación a la evolución, el estudio ACCORD mostró la evidencia más conclusiva de NAC como predictora independiente de mortalidad: más de 8,000 par- ticipantes con DM2 y el índice de riesgo para la mortalidad por todas las causas y por enfermedad cardiovascular (ECV) y NAC presente fue 2.14, después de controlar para todos los factores de riesgo de ECV tradicionales y otros, incluyendo el uso de varias clases de medicamentos ${ }^{5}$.

La intensificación del control glucémico y de la presión arterial (PA) pueden aumentar el riesgo de evento cardiovascular en personas con $\mathrm{NAC}^{19-23}$; evidencias recientes muestran asociación con variabilidad de la glucemia9,24. Además, la NAC es un factor de riesgo para la progresión de la retinopatía, la enfermedad renal diabética (DRD), la ECV y la apnea del sueno ${ }^{1,25-28}$, y la prevalencia de NAC es mayor en pacientes con las complicaciones crónicas de la DM, por tanto, se recomienda buscar síntomas y signos de NAC si hay retinopatía diabética, la DRD es también polineuropatía sensoriomotora (PNSM) ${ }^{1-3}$. Por 
otro lado, al observarse síntomas y signos de NAC en un paciente deben realizarse pruebas que excluyan otras comorbilidades que afecten e interaccionen con fármacos que pueden imitar los síntomas y signos de la $\mathrm{NAC}^{2}$.

En sus estadios iniciales, la NAC puede ser asintomática o manifestarse con síntomas de otras disautonomías de tipo gastrointestinal o genitourinario, y es detectada apenas por pruebas que muestran diminución de la variabilidad de la frecuencia cardíaca (FC) al evaluar la respiración profunda'. Los síntomas más comunes aparecen en pacientes con NAC avanzada expresada en ortostatismo: mareos, debilidad, palpitaciones, debilidad y síncope ${ }^{1-3}$, pero la correlación de estos síntomas con déficits autonómicos global es leve ${ }^{19,29}$ y aparece tardíamente en el curso de la enfermedad15-17,19,29. Debe sospecharse de NAC en pacientes con hipoglucemia inadvertida ${ }^{30}$, historia de hipoglucemia conocida y en pacientes que tuvieron episodios de hipoglucemia recurrente anterior ${ }^{31}$.

En el examen objetivo, la primera manifestación de la NAC por lo general es la taquicardia en reposo (afectación de la actividad del nervio simpático cardíaco sin oposición) e intolerancia al ejercicio ${ }^{1-3}$. En casos avanzados de NAC, esta se manifiesta con una FC fija'1. La intolerancia al ejercicio en la NAC se debe al insuficiente aumento del débito cardíaco, debido a la falta de modulación simpática ${ }^{1}$ y también hay una respuesta cardiovascular reducida a estrés fisiológico, como cirugía o infección ${ }^{32,33}$, y asociación entre NAC y arritmias cardíacas debido a alteraciones en el intervalo $\mathrm{QT}^{34}$. Las alteraciones en el equilibrio entre los sistemas simpático y parasimpático también son consideradas como proarritmogénicas ${ }^{35}$ y el tejido miocárdico denervado que tiene áreas focales de reinervación puede estar en mayor riesgo de $\operatorname{arritmia}^{36}$.
La enfermedad avanzada se asocia a HO, caída de la presión sanguínea sistólica o diastólica de $20 \mathrm{mmHg}$ o $10 \mathrm{mmHg}$, respectivamente ${ }^{1-3}$, uno a tres minutos después de asumir la ortostática, sin aumento compensatorio de la $\mathrm{FC}^{1-3,37,38}$, y es consecuencia de la denervación simpática vasomotora eferente, que causa reducción de la vasoconstricción del lecho vascular esplénico y periférico39.

Cuando es posible detectar síntomas y signos de NAC, incluido el compromiso de la variación de la FC y la presencia de $\mathrm{HO}$ en pacientes sintomáticos que presentan taquicardia en reposo e historia de control glucémico inadecuado, no es necesario realizar test adicionales para documentar la NAC, teniendo en cuenta el costo de estos, aunque siempre es importante excluir otras comorbilidades o efectos/interacciones de fármacos que pueden mimetizarla $^{1,30}$. Las tablas 3 y 4 contienen los test diagnósticos de NAC.

\section{IMPLICACIONES CLÍNICAS Y DE DIAGNÓSTICO}

La NAC se caracteriza por la denervación total o parcial del sistema cardiovascular y lo característico de esta afectación es la pérdida de la variabilidad de la FC en respuesta a distintos estímulos. Su presencia expone al individuo a un riesgo elevado por el incremento de arritmias cardíacas, infarto silente e incluso la posibilidad de sufrir muerte súbita'.

En general, la NAC puede ser asintomática y es necesario detectar signos que puedan alertar sobre su existencia, como la taquicardia de reposo. La hipotensión postural y la falta de dolor en el curso de un infarto son manifestaciones de daño avanzado y evidencian la progresividad de la lesión del SNA; otra es la baja tolerancia al ejercicio. Su presencia representa un mayor riesgo quirúrgico y 
TABla 3. Algoritmo para el diagnóstico de la taquicardia de reposo

\begin{tabular}{|c|c|c|}
\hline Síntomas & $\begin{array}{l}\text { Signos/test } \\
\text { diagnósticos }\end{array}$ & Diagnóstico diferencial \\
\hline Palpitaciones & $\begin{array}{l}\text { Examen clínico: } \\
\text { FC de reposo } \\
>100 \mathrm{lpm}\end{array}$ & $\begin{array}{l}\text { Anemia } \\
\text { Hipotiroidismo } \\
\text { Fiebre } \\
\text { ECV (fibrilación auricular, } \\
\text { flutter, otras) } \\
\text { Deshidratación } \\
\text { Insuficiencia adrenal } \\
\text { Medicaciones } \\
\text { Agentes } \\
\text { simpaticomiméticos } \\
\text { (asma) } \\
\text { Dosis altas de agentes } \\
\text { para gripe con efedrina o } \\
\text { pseudoefedrina } \\
\text { Suplementos dietéticos } \\
\text { Tabaquismo, uso de } \\
\text { alcohol, cafeína } \\
\text { Drogas ilícitas (cocaína, } \\
\text { anfetaminas, } \\
\text { metanfetamina) }\end{array}$ \\
\hline
\end{tabular}

anestésico; además, se vincula a una menor supervivencia postinfarto ${ }^{1-3}$.

El diagnóstico en etapas tempranas se obtiene mediante la aplicación de test que permiten evaluar la respuesta de la funcionalidad cardíaca ante ciertos estímulos. Ewing ${ }^{40}$ describió cinco pruebas, de las cuales tres observan la respuesta parasimpática con valoración del espacio R-R del electroencefalograma (EEG) ante la respiración profunda (seis ritmos respiratorios por minuto), la maniobra de
TABLA 4. Algoritmo diagnóstico para la hipotensión postural

\begin{tabular}{|c|c|c|}
\hline Síntomas & $\begin{array}{l}\text { Signos/test } \\
\text { diagnósticos }\end{array}$ & Diagnóstico diferencial \\
\hline $\begin{array}{l}\text { Mareos } \\
\text { Debilidad } \\
\text { Desmayos } \\
\text { Alteraciones } \\
\text { visuales } \\
\text { Síncope }\end{array}$ & $\begin{array}{l}\text { Reducción de } \\
20 \text { mmHg en } \\
\text { PAS } \\
\text { o } \\
\text { Reducción de } \\
10 \text { mmHg en } \\
\text { PAD }\end{array}$ & $\begin{array}{l}\text { Insuficiencia adrenal } \\
\text { Depleción de volumen } \\
\text { intravascular } \\
\text { Pérdida sanguínea, anemia } \\
\text { aguda o deshidratación } \\
\text { Gestación, posparto } \\
\text { ECV } \\
\text { Arritmias, insuficiencia } \\
\text { cardíaca o miocárdica } \\
\text { Pericárdica, enfermedad } \\
\text { cardíaca valvular } \\
\text { Alcohol } \\
\text { Medicaciones: } \\
\text { antiadrenérgicos, } \\
\text { antianginosos, } \\
\text { antiarrítmicos, } \\
\text { anticolinérgicos, } \\
\text { diuréticos, } \\
\text { IECA, bloqueadores del } \\
\text { receptor de la } \\
\text { angiotensina, narcóticos, } \\
\text { neurolépticos y sedativos }\end{array}$ \\
\hline
\end{tabular}

ECV: enfermedad cardiovascular; IECA: inhibidores de la enzima convertidora de angiotensina; PAD: presión arterial diastólica; PAS: presión arterial sistólica.

Valsalva (espiración forzada con la glotis cerrada) y el cambio de decúbito supino a la postura de pie (Tabla 5).

Para constatar la integridad o alteración de la actividad autónoma con predominio del componente parasimpático es necesario realizar un electrocardiograma continuo, o contar con un software especial que permita la automatización en los análisis

TABLA 5. Pruebas cardiovasculares. Test de Ewing

\begin{tabular}{|c|c|c|c|}
\hline & Normal & Dudosa & Anormal \\
\hline Maniobra de Valsalva & 1.21 o más & - & 1.20 o menos \\
\hline Respiración profunda & 15 latidos o más & 11 a 14 latidos & 10 latidos o menos \\
\hline Cambio de decúbito & 1.04 o mayor & 1.01 a 1.03 & 1.00 o menor \\
\hline Hipotensión postural & $10 \mathrm{mmHg}$ & 10 a $29 \mathrm{mmHg}$ & 30 mmHg o más \\
\hline Hand grip & 15 mmHg o más & 11 a $14 \mathrm{mmHg}$ & $10 \mathrm{mmHg}$ o menos \\
\hline
\end{tabular}

Adaptada de Ewing, et al., $1980^{40}$. 
correspondientes. Las otras dos son maniobras para analizar la respuesta predominantemente simpática del arco reflejo y comprenden maniobras clínicas: la medición de la PA con los cambios de posición (en búsqueda de la caída de la tensión sistólica ante la modificación postural) y la prueba de presión sobre un hand grip (o resorte calibrado cuyo apretón tiene que provocar la elevación de la presión arterial diastólica [PAD] $)^{1,3}$. Los consensos de las conferencias de San Antonio en 1988 y la segunda conferencia en 1992, auspiciadas por la American Diabetes Association (ADA) y la American Academy of Neurology (AAN), pusieron énfasis en la estandarización de estas pruebas diagnósticas, el seguimiento con estos test evalúa la progresión de la NAC en su totalidad, como muestra la tabla $6^{41}$.

Un aspecto que considerar es la medición del segmento QT, dada la relación entre muerte y la prolongación de este en el ECG. En el 23\% de los pacientes con segmento QT prolongado se registró una muerte súbita dentro de los tres años desde el momento en que se realizó el diagnóstico ${ }^{42}$. El valor normal del segmento QT corregido (QTC) es $\leq$ $440 \mathrm{~ms}$. Otro examen que aporta datos de valor es el análisis espectral de la FC; se obtiene por medio de un sistema computarizado y permite determinar la relación de la actividad simpática/parasimpática del corazón en condiciones de reposo, obteniéndose información sobre el estado del SNA cardíaco ${ }^{1,3}$.

La hipotensión postural en ortostatismo que se presenta ante los cambios de posición puede manifestarse con mareos e incluso síncopes y es la resultante del déficit de la respuesta vasoconstrictora por lesión de las fibras posgangliónicas responsables de la vasoconstricción vascular. Es característico el descenso de más de 20 mmHg de PA sin taquicardia compensadora con las modificaciones de decúbito. Los cuadros sincopales se pueden confundir con hipoglucemias o síndrome vertiginoso y hay
TABLA 6. Interpretación de los resultados de las pruebas cardiovasculares de Ewing y la ADA/AAN

\begin{tabular}{|l|l|}
\hline Estadio temprano & $\begin{array}{c}\text { Anormalidad de la variabilidad de la } \\
\text { FC durante el test de la respiración } \\
\text { profunda solamente }\end{array}$ \\
\hline Estadio intermedio & \begin{tabular}{|l} 
Anormalidad de la variabilidad de la \\
FC ante la maniobra de Valsalva
\end{tabular} \\
\hline Estadio severo & La presencia de hipotensión postural \\
\hline Diagnóstico & $\begin{array}{l}\text { La sola presencia de síntomas no } \\
\text { hace diagnóstico } \\
\text { La presencia de } 2 \text { o más pruebas de } \\
\text { las } 5 \text { de Ewing alteradas determina } \\
\text { el diagnóstico de NAC }\end{array}$ \\
\hline Indicación clínica & $\begin{array}{l}\text { Pacientes con historia de mal control } \\
\text { metabólico } \\
\text { Pacientes con DM2 desde el } \\
\text { diagnóstico } \\
\text { A los } 5 \text { años del diagnóstico de DM1 } \\
\text { Pacientes con otros factores de } \\
\text { riesgo de ND (hipertensión arterial, } \\
\text { tabaquismo, dislipidemia) } \\
\text { Pacientes con microalbuminuria } \\
\text { Pacientes con NAD en otros } \\
\text { territorios } \\
\text { Pacientes con PNSD }\end{array}$ \\
\hline Recomendaciones & $\begin{array}{l}\text { La sola presencia de síntomas no } \\
\text { hace diagnóstico } \\
\text { Los test propuestos deben realizarse } \\
\text { para establecer el diagnóstico } \\
\text { Deberán usarse pruebas que evalúen } \\
\text { tanto el parasimpático como el } \\
\text { simpático } \\
\text { La batería completa de test debería } \\
\text { realizarse para el seguimiento y } \\
\text { evaluación del deterioro en los } \\
\text { pacientes diagnosticados } \\
\text { La repetición de los test debe } \\
\text { realizarse anualmente }\end{array}$ \\
\hline
\end{tabular}

AAN: American Academy of Neurology; ADA: American Autonomic Society; DM: diabetes mellitus; FC: frecuencia cardíaca; HTA: hipertensión arterial; NAC: neuropatía autonómica cardiovascular; ND: neuropatía diabética; NAD: neuropatía autonómica diabética; PNSD: polineuropatía simétrica distal. Adaptada de Ewing, et al., $1980^{40}$ y ADA/AAN, $1996^{42}$.

fármacos que agravan la situación como los antidepresivos tricíclicos y los diuréticos ${ }^{37}$.

\section{SITUACIONES ESPECIALES: INFARTO E ISQUEMIA SILENTE}

El infarto indoloro o silente, que puede presentarse en alrededor del $33 \%$ de las personas con diabetes, 
solo se descubre en los pacientes por medio de un examen de rutina o en otros contextos alejados de la pesquisa cardiovascular. La prolongación del tiempo entre el comienzo de la isquemia miocárdica y la percepción del dolor puede resultar de la afectación del plexo subepicárdico y puede explicar la ausencia de dolor por falta de transmisión del impulso ${ }^{1,3}$.

El infarto silente en pacientes con DM ha sido atribuido a la presencia de daño simpático aferente en las fibras que inervan el miocardio. La existencia de isquemia silente en sujetos sin DM y con DM sin NAC, así como la variabilidad intrasujeto en la percepción del dolor anginoso, sugieren la posibilidad de otros factores no neuropáticos en la génesis de la isquemia indolora, como las alteraciones en los mecanismos centrales para la percepción del dolor. En estos enfermos se debería realizar, mediante estudios de ECG de esfuerzo u otros métodos no invasivos, la detección temprana y tratamiento de la enfermedad coronaria isquémica ${ }^{29}$.

Los estudios con técnicas de radionucleótidos se han utilizado para cuantificar de forma directa la inervación simpática del corazón. Dichos estudios están fundamentados en que la estructura neuronal en el miocardio ventricular está constituida primariamente por fibras nerviosas simpáticas, que viajan a través de la superficie subepicárdica, siguiendo principalmente a los vasos coronarios y, como estos, progresan desde la base del corazón al ápex, penetran gradualmente al miocardio e inervan el endocardio por los plexos terminales. Dentro de los radionucleótidos, el metayodobenzilguanidina (MIBG), un análogo no metabolizable de la norepinefrina, participa en la captación de este en las neuronas simpáticas posganglionares. Existe evidencia de la disminución de la captación de MIBG en el miocardio en pacientes con NAC diagnosticados por pruebas de función autonómica ${ }^{11}$.
La asociación entre NAC e isquemia silente tiene gran importancia para la clínica diaria, ya que esta reduce o afecta al dolor isquémico, y el paciente no puede reconocer en forma oportuna los síntomas de la isquemia miocárdica o del infarto, retrasándose con ello el tratamiento ${ }^{1-3}$. En definitiva, se plantean como conductas necesarias la sospecha y búsqueda de la NAC en todas las personas con DM que cumplan con los criterios antes mencionados con el objetivo de alertarlas y poder intervenir adecuadamente.

\section{TRATAMIENTO ACTUAL DE LA NEUROPATÍA AUTONÓMICA CARDIOVASCULAR}

Varias revisiones de la literatura'-3,43 enfatizan que la NAC es una de las complicaciones de la DM menos diagnosticadas y, consecuentemente, subtratada, aunque sea considerada en la actualidad un marcador de riesgo para macroangiopatía ${ }^{1,4}$ y es un factor de mal pronóstico para microangiopatía ${ }^{22}$. Otros trabajos sobre tratamiento de la NAC apuntan que en sus fases iniciales y subclínicas aún puede prevenirse, estabilizarse y mejorarse, por eso en los casos de NAC avanzada o grave apenas queda el tratamiento sintomático ${ }^{44,45}$. Por lo tanto, el tratamiento de la NAC puede ser dividido en dos fases: preventivo (fases subclínicas) y sintomático (fases avanzadas).

\section{TRATAMIENTO PREVENTIVO DE LA NEUROPATÍA AUTONÓMICA CARDIOVASCULAR}

Su objetivo es la prevención de la instalación de la NAC o su progresión. Hay cinco factores de riesgo modificables, cuyo abordaje va dirigido a objetivos específicos y preestablecidos, resultando en una 
disminución del $63 \%$ en la progresión y/o instalación de la NAC ${ }^{46}$ : hiperglucemia, tabaquismo, HTA sistólica, dislipidemia y microalbuminuria. El abordaje multifactorial recomendado se expone en la tabla $7^{47}$.

Otra medida, dentro de un programa de actividades, es mejorar la condición física de forma escalonada y adaptada a la respuesta y tolerancia del paciente. Estas medidas pueden y deben ser recomendadas, pues hay evidencias que demuestran que mejoran tanto la NAC inicial como los estadios más graves ${ }^{48}$. Es prudente, sin embargo, realizar previamente un test ergométrico en estos pacientes ${ }^{3}$.

\section{TRATAMIENTO SINTOMÁTICO DE LA NEUROPATÍA AUTONÓMICA CARDIOVASCULAR}

El tratamiento sintomático de la NAC está indicado en los pacientes con $\mathrm{HO}$ o con taquicardia de reposo (FC > $100 \mathrm{lpm})$.

Al diagnosticar la taquicardia de reposo, deben utilizarse preferentemente los betabloqueadores selectivos y liposolubles como el metoprolol en dosis de 25 a $100 \mathrm{mg}$ o el nebivolol en dosis de 2.5 a $5.0 \mathrm{mg}$ administrados de preferencia una vez al día y por la noche. El tratamiento de la HO contempla medidas no farmacológicas y farmacológicas.

\section{Tratamiento no farmacológico de la hipotensión ortostática}

Debe ser siempre la primera opción y hay cinco medidas secuenciales eficientes, seguras y con bajo costo que deben ser tomadas en cuenta:
TABLA 7. Metas utilizadas en el estudio Steno 2 para abordaje multifactorial de la diabetes prevención de NAC

\begin{tabular}{|l|c|c|}
\hline Tratamiento & Patrón & Intensivo \\
\hline PAS (mmHg) & $<160$ & $<140$ \\
\hline PAD (mmHg) & $<95$ & $<85$ \\
\hline HbA1c (\%) & $<7.5$ & $<6.5$ \\
\hline Triglicéridos (mg/dl) & $<194$ & $<150$ \\
\hline Colesterol total (mg/dl) & $<250$ & $<193$ \\
\hline Colesterol HDL (mg/dl) & $>35$ & $>42$ \\
\hline $\begin{array}{l}\text { Uso de IECA } \\
\text { independientemente } \\
\text { de la presión arterial }\end{array}$ & Sí & Sí \\
\hline $\begin{array}{l}\text { Uso de aspirina en pacientes } \\
\text { con isquemia }\end{array}$ & No & Sí \\
\hline $\begin{array}{l}\text { Uso de aspirina en pacientes con } \\
\text { enfermedad arterial periférica }\end{array}$ & & \\
\hline
\end{tabular}

HDL: colesterol de la lipoproteina de alta densidad; IECA: inhibidores de la enzima convertidora de angiotensina; NAC: neuropatía autonómica cardiovascular; PAD: presión arterial diastólica; PAS: presión arterial sistólica. Adaptada de Gaede, et al., $1999^{47}$.

- Revisar y de ser necesario retirar fármacos cardiovasculares (antihipertensivos y vasodilatadores) y los fármacos de acción sobre el sistema nervioso central (antidepresivos tricíclicos y ansiolíticos). Si no es posible eliminar un fármaco específico, este deberá ser administrado al acostarse para reducir la HO durante el día. Los fármacos antihipertensivos de media vida corta, como el captopril, el diltiazem o el verapamilo, son preferibles y pueden ser administrados también en el momento de acostarse.

Los pacientes deben ser alertados sobre los riesgos de caídas, sobre todo al levantarse en la noche y las medidas de seguridad para evitar accidentes imprevistos que deben ser consideradas son:

- Elevar la cabecera de la cama durante la noche $(10$ a $30 \mathrm{~cm})$. En realidad, es suficiente con utilizar más de una almohada para dormir. 
- Aconsejar al paciente levantarse de la posición acostada (decúbito dorsal) de forma lenta y gradualmente, esto es, el paciente deberá sentarse lentamente primero y hacer la dorsiflexión de los pies.

- Informar al paciente qué ejercicios posturales, como cruzar las piernas después de levantarse, pueden aumentar el débito cardíaco y la PA en hasta un $16 \%{ }^{1,43}$.

- Aumentar la ingestión de sodio y agua, si no hubiera contraindicación (como insuficiencia cardíaca congestiva [ICC]) y uremia.

- Aunque se ha preconizado el uso de medias elásticas para incrementar el tono vascular periférico, esta medida suele ser ineficiente, pues se ha comprobado que no aumenta el tono vascular esplénico (más importante en la fisiopatología de la $\mathrm{HO}$ en individuos con DM). Además, la presencia de enfermedad arterial periférica, presente en el $50 \%$ de los pacientes ${ }^{49}$, contraindica formalmente el uso de medias compresivas en los miembros inferiores.

\section{Tratamiento farmacológico de la hipotensión ortostática}

Si las medidas no farmacológicas no resultan eficaces, hay cuatro fármacos recomendados para el tratamiento de la HO sintomática3 ${ }^{3}$ que son:

- Fludrocortisona (0.1 y $0.05 \mathrm{mg}$ ), dosis de $0.1 \mathrm{a}$ $0.4 \mathrm{mg} /$ día. En adición debe aconsejarse una dieta rica en sodio, que resulta eficaz en los casos más graves. Se debe estar atentos para diagnosticar oportunamente los efectos colaterales que pueden aparecer, como hipertensión arterial supina, edema periférico y empeoramiento de la ICC.
- Cafeína (100 mg en la forma de citrato o anhidra), eficaz en dosis de $100 \mathrm{mg}$ cada 8 horas.

- Midodrina (5 y $10 \mathrm{mg}$ ). Es un potente agonista alfaadrenérgico con propiedades vasoconstrictoras. Se debe vigilar el empeoramiento de la hipertensión arterial supina y no debe ser administrado después de las 18:00 h. Una dosis eficaz y recomendable en los casos refractarios puede esquematizarse así: $10 \mathrm{mg}$ las 7:00 h (mañana), $5.0 \mathrm{mg}$ a las $12: 00 \mathrm{~h}$ (medio día) y $2.5 \mathrm{mg}$ a las 17:00 h (tarde).

- Eritropoyetina (ámpulas de 1,000, 2,000, 3,000 y 4,000 unidades). Está indicada en casos de anemia $(-<12 \mathrm{~g} / \mathrm{dl})$ en pacientes con enfermedad renal crónica (IRC) estadios IV y V. La dosis total preconizada para HO varía entre 50 y 60 unidades por kg de peso, divididas en tres dosis por semana, vía subcutánea (s.c.), durante dos a cuatro meses. En los pacientes con ICC e IRC se debe administrar la dosis total una vez por semana (6o U/kg peso) hasta cuatro meses. En los pacientes con cifras de hemoglobina por encima de $12 \mathrm{~g} / \mathrm{dl}$ los riesgos son mayores para desarroIlar ICC y AVC.

Aunque el análogo de la somatostatina octreótido (50 mcg tres veces al día, s.c.) ha sido sugerido en los casos refractarios de $\mathrm{HO}$, los efectos adversos como diarrea y descontrol glucémico limitan su recomendación.

\section{RECOMENDACIONES CON BASE EN EL GRADO DE EVIDENCIAS ${ }^{3}$}

- Los síntomas y signos de neuropatía autonómica deben ser evaluados en pacientes con complicaciones microvasculares y neuropáticas. E 
- En la presencia de síntomas y signos de NAC, los test que excluyen otras comorbilidades o efectos de fármacos e interacciones que imitan NAC deben realizarse. $\mathbf{E}$

- Considerar evaluar los síntomas y signos de NAC en pacientes con hipoglucemias desconocidas. $\mathbf{C}$

\section{NEUROPATÍA AUTONÓMICA GASTROINTESTINAL}

El compromiso autonómico puede involucrar cualquier porción del tracto gastrointestinal con manifestaciones que incluyen la dismotilidad esofágica, la gastroparesia, el retardo en el vaciamiento gástrico, la constipación, la diarrea y la incontinencia fecal. Hay que resaltar que se trata de una alteración de evolución crónica, pero teniendo presente que en ocasiones la hiperglucemia aguda enlentece el vaciamiento gástrico y la hipoglucemia lo acelera. La prevalencia es limitada, pues los informes fueron casos seleccionados más que grandes poblaciones estudiadas. En el único estudio con base poblacional en 10 años se mostró un $5 \%$ en pacientes con DM1, mientras en DM2 solamente un 1\% en relación con los sujetos controles ${ }^{50}$. El transito esofágico retrasado en el $50 \%$ de pacientes con larga evolución de diabetes puede estar asociado a regurgitación, disfagia y propensión a la erosión esofágica inducida por estenosis pilórica. La gastroparestesia afecta al $40 \%$ de pacientes con larga evolución de diabetes ${ }^{1}$.

Los síntomas son variables y más comunes en pacientes con peor control crónico de la glucemia y desorden psicológico ${ }^{51}$. Sin embargo, puede ser silente en la mayoría de los casos, no hay correlación con la severidad de la gastroparesia y está pobremente relacionado con el vaciamiento gástrico ${ }^{1-3}$.
Los síntomas asociados al retardo del vaciamiento gástrico son: saciedad temprana, anorexia, distensión, náusea, vómito, dispepsia, dolor abdominal y pérdida de peso inespecífica. Estos síntomas son similares a otras condiciones y pueden apenas estar asociados a la presencia de la diabetes ${ }^{3}$. Siempre se deberán excluir otras causas de gastroparesia o enfermedad gástrica ${ }^{1,3}$.

Es importante recordar la relación entre hiperglucemia, hipoglucemia y cambios agudos de la glucemia con la alteración del vaciamiento gástrico, que afecta directamente el control de la glucemia, así como variabilidad glucemia o hipoglucemias inexplicables debido a la disociación entre la absorción de la ingesta de alimentos y el perfil farmacocinético de la insulina y otros fármacos ${ }^{3}$. Los síntomas intestinales de diarreas acuosas con sobrecrecimiento bacteriano y estreñimiento se asocian con alteraciones de la absorción, mal control metabólico, malnutrición, regulación posprandial anormal de la tensión arterial, empobrecimiento de la QoL y elevadas tasas de hospitalización. En ocasiones se puede evidenciar pérdida de control en el esfínter anal interno y referir episodios recurrentes de hipo por vaciamiento gástrico deficiente. Las diarreas son usualmente intermitentes, bruscas, nocturnas con ruidos intestinales en el abdomen inferior e incontinencia fecal².

Las obstrucciones mecánicas y la enfermedad péptica deben ser excluidas con esofagogastroduodenoscopia y, de ser necesario, considerar el estudio del estómago con bario. El retardo del vaciamiento gástrico debe ser documentado y el estándar de oro para esto lo da la medida del vaciamiento de elementos sólidos cada 15 minutos durante cuatro horas mediante la evaluación del centelleo gama (tecnecio 99m) o el uso de ácido octanoico respirado ${ }^{1-3}$. 
Deben excluirse la diarrea diabética, la enfermedad celíaca, la insuficiencia pancreática exocrina y el sobrecrecimiento bacteriano en el intestino delgado. Los test de evaluación del esfínter del músculo motor anorrectal y la función sensorial deben ser considerados en su uso clínico'. El tratamiento de la gastroparesia es un verdadero desafío médico. Los cambios en el régimen de dieta considerando ingestas pequeñas son importantes, disminuyendo adicionalmente la grasa y la calidad de la alimentación ${ }^{1-3}$. Se deberán suspender los fármacos que puedan alterar la motilidad gastrointestinal, como opioides, anticolinérgicos, tricíclicos y agonistas del receptor del péptido similar al glucagón tipo 1 (GLP-1) 3 .

El único fármaco aprobado por la FDA (Food and Drug Administration) y la EMA (European Medical Agency) es la metoclopramida, a pesar de que la evidencia es pequeña en relación con la mejoría de los síntomas y tiene la posibilidad de provocar efectos extrapiramidales. Es por ello por lo que no se recomienda su utilización más allá de cinco días ${ }^{1,3}$.

\section{RECOMENDACIONES CON BASE EN EL GRADO DE EVIDENCIAS ${ }^{52}$}

- Evaluar la gastroparesia en personas con ND, retinopatía y/o nefropatía mediante la evaluación de los síntomas de variabilidad glucémica inesperada, saciedad precoz, hinchazón, náuseas y vómitos. C

- Exclusión de otras causas documentadas para alterar el vaciado gástrico, como antes de realizar pruebas especializadas para la gastroparesia es necesario el uso de opioides o agonistas del receptor del GLP-1 y obstrucción de la salida gástrica orgánica. C
- Para evaluar la gastroparesia, medir el vaciado gástrico con gammagrafía de sólidos digeribles a intervalos de 15 minutos durante $4 \mathrm{~h}$ después de la ingesta de alimentos o usar una prueba de aliento con ácido 13 C-octanoico. B

- Considerar el uso de metoclopramida durante un tiempo corto (hasta cinco días) en dosis $10 \mathrm{mg} 30 \mathrm{~min}$ antes de las comidas, porque sus efectos extrapiramidales sobrepasan los beneficios. $E$

En América Latina, se recomiendan otros fármacos y medidas $^{2}$ para tratar la gastroparesia²:

- Domperidona. Dosis normal, 10 a $20 \mathrm{mg}$ oral tres veces al día, 30 a 60 min antes de las comidas.

- Eritromicina. Dosis normal, $500 \mathrm{mg}$ oral tres veces al día, durante 7 a 10 días. Se debe administrar 30 minutos antes de las comidas.

Para las diarreas, las recomendaciones son²:

- Ingesta de fibras solubles en dieta: 20 a 30 g/día.

- Antibióticos (2 a 3 semanas): tetraciclina 250$300 \mathrm{mg}$ una vez/día; ciprofloxacino $500 \mathrm{mg}$ dos veces/dia, trimetoprina + sulfametoxazol F $800 \mathrm{mg}$ $+160 \mathrm{mg}$ dos veces/dia.

- Ingesta de fibra en dieta: 20 a 30 g/día.

- Laxantes de fibra: metamucil, polietilenglicol o lactulosa.

- Clonidina. Dosis normal: 0.1 mg dos veces al día.

- Antiadiarreicos (loperamida), anticolinérgicos (escopolamina, homatropina, codeína, atropina), octreotide. 


\section{NEUROPATÍA AUTONÓMICA UROGENITAL}

La NAD puede también causar alteraciones genitourinarias, incluyendo la vejiga y la disfunción sexual ${ }^{1,52}$. Su prevalencia exacta y la patogénesis detallada aún no se han investigado y estudiado en ensayos clínicos o básicos multicéntricos, a gran escala o aleatorizados, por ello es necesario realizar un estudio validado y estandarizado para mejorar el tratamiento de la cistopatía diabética en la práctica clínica53.

\section{DISAUTONOMÍA VESICAL}

Se estima que la prevalencia es del $43-87 \%$ en la DM1 y del $25 \%$ en la DM2, la duración de la DM, la retinopatía y la PNSD están asociadas con la severidad de la disfunción vesical y la incontinencia; la correlación con la PNSD varía del 75 al 100\%1,52. La cistopatía diabética es multifactorial, incluidas las alteraciones del músculo detrusor, la disfunción neuronal, el urotelio y la uretra1-3,52. Los síntomas clásicos de la cistopatía diabética son: disminución de la sensación de la vejiga, aumento de la capacidad de la vejiga y deterioro del vaciado de la vejiga con el consiguiente aumento del volumen residual posvacío; sin embargo, la evidencia clínica reciente indica también la presencia de síntomas de almacenamiento y síntomas de vejiga hiperactiva53. Es por esta razón que los individuos con DM y disfunción de la vejiga están más predispuestos al desarrollo de las infecciones de las vías urinarias (IVU), incluyendo la pielonefritis, que pueden acelerar o exacerbar la insuficiencia renal².

En mujeres, la incontinencia vesical es aumentada $50 \%$ en las de media y más edad, resultando en estrés, limitaciones de las actividades diarias y pobre
QoL. En el estudio de las enfermeras (Nurses' Health Study), el riesgo estaba aumentado en un 30-100\%, además, las mujeres con DM están más predispuestas a desarrollar incontinencia urinaria grave y sintomática: urgencia y pérdida involuntaria de orina, y las tratadas con insulina tienen riesgo más elevado que las tratadas con fármacos orales ${ }^{54}$.

En los hombres, las IVU son comunes y atribuidas a hiperplasia benigna de la próstata (HBP). Los hombres con HBP y DM tienen más IVU que los que no tienen $D^{54}$. Los efectos de la DM en las IVU han sido más estudiados en la DM1 que en la DM2. En el DCCT/EDIC, la IVU moderada a grave se reportó en el 20\% después 10 años de seguimiento y 22.1 de duración de la DM55.

La elección del tratamiento depende de los síntomas clínicos y las anomalías urodinámicas, por lo cual se hace necesario descartar patologías concurrentes prostáticas y del aparato ginecológico, y tener presente que fármacos como la amitriptilina y otros propician las alteraciones de la dinámica urinaria. La evaluación urodinámica es la piedra angular del diagnóstico y determina las estrategias de tratamiento ${ }^{1-3,52}$. Esto puede incluir cistometría, uroflujo, estudios simultáneos de presión/flujo, electromiografía de esfínteres y perfilometría de presión uretral o evaluación de presiones de punto de fuga. Además, las pruebas electrofisiológicas u otras evaluaciones pueden ser útiles para la evaluación de la neuropatía periférica ${ }^{1,52}$.

El cuestionario mejor validado es el Lower urinary tract symptoms y otros cuestionarios de QoL son importantes ${ }^{52}$. Los exámenes estandarizados sirven para cuantificar la tasa de flujo urinario pico y el volumen urinario residual posvaciado valorados mediante ecografía del sistema urinario. Se observa afectación de la capacidad cistométrica con descenso de la contractilidad del detrusor. Los pacientes 
con insuficiencia renal moderada a severa tienen un alto riesgo de padecer IVU graves, disfunción eréctil (ED) y disfunciones sexuales simultáneas ${ }^{1,2}$. La evaluación de la sensación en la región perineal, del reflejo bulbocavernoso y del tono esfinteriano puede ayudar a identificar ND periférica. Debe realizarse un examen uroginecológico para excluir prolapsos y otros desórdenes pélvicos.

\section{DISFUNCIÓN ERÉCTIL}

De intensidad moderada a severa, la ED está presente en el 5 al 20\% de los hombres diabéticos. La ED con o sin eyaculación retrógrada es tres veces más frecuente en pacientes con DM frente a sin $D^{3-4}$. La etiología es multifactorial; la ED comparte factores de riesgo con las ECV, como hipertensión arterial, dislipidemia, obesidad, tabaquismo, medicación y factores psicológicos ${ }^{52}$.

Hay evidencia de asociaciones entre la ED y otras complicaciones de la diabetes, incluyendo la NAC y la cardiopatía isquémica ${ }^{1-3,56}$. La ED puede ser una consecuencia de la neuropatía autonómica, ya que la neurotransmisión autónoma controla el tono y la función del músculo liso cavernoso y el detrusor ${ }^{56,57}$. Se debe hacer el diagnóstico después de establecer los signos y síntomas de ED y después de excluir las causas alternativas. Los médicos deben considerar realizar una evaluación hormonal (hormona luteinizante, testosterona, testosterona libre, prolactina) para descartar hipogonadismo. Además, deben excluirse varios medicamentos y causas orgánicas ${ }^{1,52}$. El control de la glucosa se ha asociado con una menor incidencia de ED en hombres con DM1, la evidencia es menos fuerte en $\mathrm{DM} 2^{1,52}$.

Este cuadro clínico conlleva un número elevado de pacientes con síndrome ansioso depresivo. El diagnóstico se basa en la historia médica y sexual los factores de riesgo y el examen físico; las pruebas de laboratorio deben adaptarse a las quejas del paciente (incluidos los cuestionarios validados, como el Índice de Función Eréctil y el Perfil de Encuentro Sexual) y se deben emplear además en aquellos pacientes que consultan por retención urinaria y/o disfunción vesical que permiten evaluar la frecuencia y severidad de la lesión ${ }^{1,52}$. El tratamiento farmacológico incluye inhibidores de la fosfodiesterasa 5 (PDE5-I) como tratamiento de primera línea y prostaglandinas transuretrales debido a su alta eficacia y seguridad en pacientes con DM, inyecciones intracavernosas, dispositivos de vacío y prótesis de pene en casos más avanzados ${ }^{1-3,52}$. La disfunción sexual femenina se expresa en mujeres con DM con mayor frecuencia que sin $\mathrm{DM}^{1-3}$ y las quejas incluyen menor deseo sexual, dolor en el coito e inadecuada lubricación.

\section{DISFUNCIÓN SUDOROMOTORA}

Se caracteriza por piel seca, anhidrosis e intolerancia al calor ${ }^{1-3}$. Una forma más rara se manifiesta con exceso de sudoración en cabeza y cuello desencadenada por la ingesta de alimentos o debido al olor de la comida y/o alteraciones gustativas. Antes descrita solamente en relación con la NAD, se la asocia también a pacientes en diálisis o $\mathrm{DRD}^{10}$.

No hay evidencia para realizar pesquisa sistemática y la sospecha clínica aparece después que los pacientes comienzan a notar hipoglucemia después de la ingesta de comida. La eficacia del tratamiento con agentes tópicos antimuscarínicos como el glicopirrolato se confirmó en un estudio aleatorizado y controlado; su aplicación atenúa los síntomas en muchos pacientes ${ }^{1-3,52}$. 


\section{RECOMENDACIONES ${ }^{1-3,52}$}

- Se debe realizar una prueba de orina posmiccional y una tira reactiva de orina (cultivo opcional) anualmente en todos los pacientes con diabetes dependiente de la insulina. $\mathbf{C}$

- El tratamiento de elección para una vejiga contráctil sigue siendo el cateterismo intermitente. $\mathbf{B}$

- Considerar el examen de hombres con otras formas de DN (PNSD y NAD) para la ED con preguntas simples sobre la libido y la capacidad de alcanzar y mantener la erección. C

- Considerar a los pacientes con otras formas de DN (PNSD y NAD) para los síntomas del tracto urinario inferior y la disfunción sexual femenina en presencia de infecciones recurrentes del tracto urinario mediante preguntas específicas sobre síntomas, como nocturia, dolor durante el coito y otros. B

- Iniciar terapia con PDE5-I en hombres que buscan tratamiento para la ED, siempre que no haya contraindicación para su uso. A

- La elección de un PDE5-I específico debe basarse en las preferencias individuales de los hombres con ED, incluida la facilidad de uso, el costo de los medicamentos y el perfil de efectos adversos. $\mathbf{C}$

- Otras opciones pueden ser útiles en pacientes que no responden a los PDE5-I. B

- No se recomienda el uso habitual de análisis de sangre hormonales o tratamiento hormonal en el tratamiento de pacientes con disfunciones eréctiles. $\mathbf{E}$
- Los pacientes con alto riesgo cardiovascular deben estabilizarse mediante tratamiento cardiológico antes de considerar la actividad sexual o se recomienda el tratamiento de la ED. A

\section{HIPOGLUCEMIA ASINTOMÁTICA O INADVERTIDA}

\author{
Puntos clave:
}

- La hipoglucemia sigue siendo la principal barrera para la realización del control glucémico a largo plazo y puede causar morbilidades graves en individuos con diabetes.

- Las hipoglucemias asintomáticas se observan más en personas con DM1 y son menos frecuentes entre aquellas con $\mathrm{DM}_{2}$, aunque las hospitalizaciones en estas últimas son más comunes.

- La exposición a la hipoglucemia frecuente y con episodios repetidos es un factor importante en la patogénesis de la disfunción autonómica asociada a la hipoglucemia (DAAH).

- Estudios clínicos muestran que las hipoglucemias asintomáticas son más comunes en individuos con DM de larga duración, que presentan hipoglucemias frecuentes, bajo control intensivo de la glucemia y entre aquellos con edad más avanzada.

- La hipoglucemia asintomática es una gran limitación para alcanzar el control efectivo de la DM y produce una reducción impactante de la QoL.

La principal barrera actualmente para lograr el control glucémico estricto es la hipoglucemia iatrogénica, causada más comúnmente por el tratamiento 


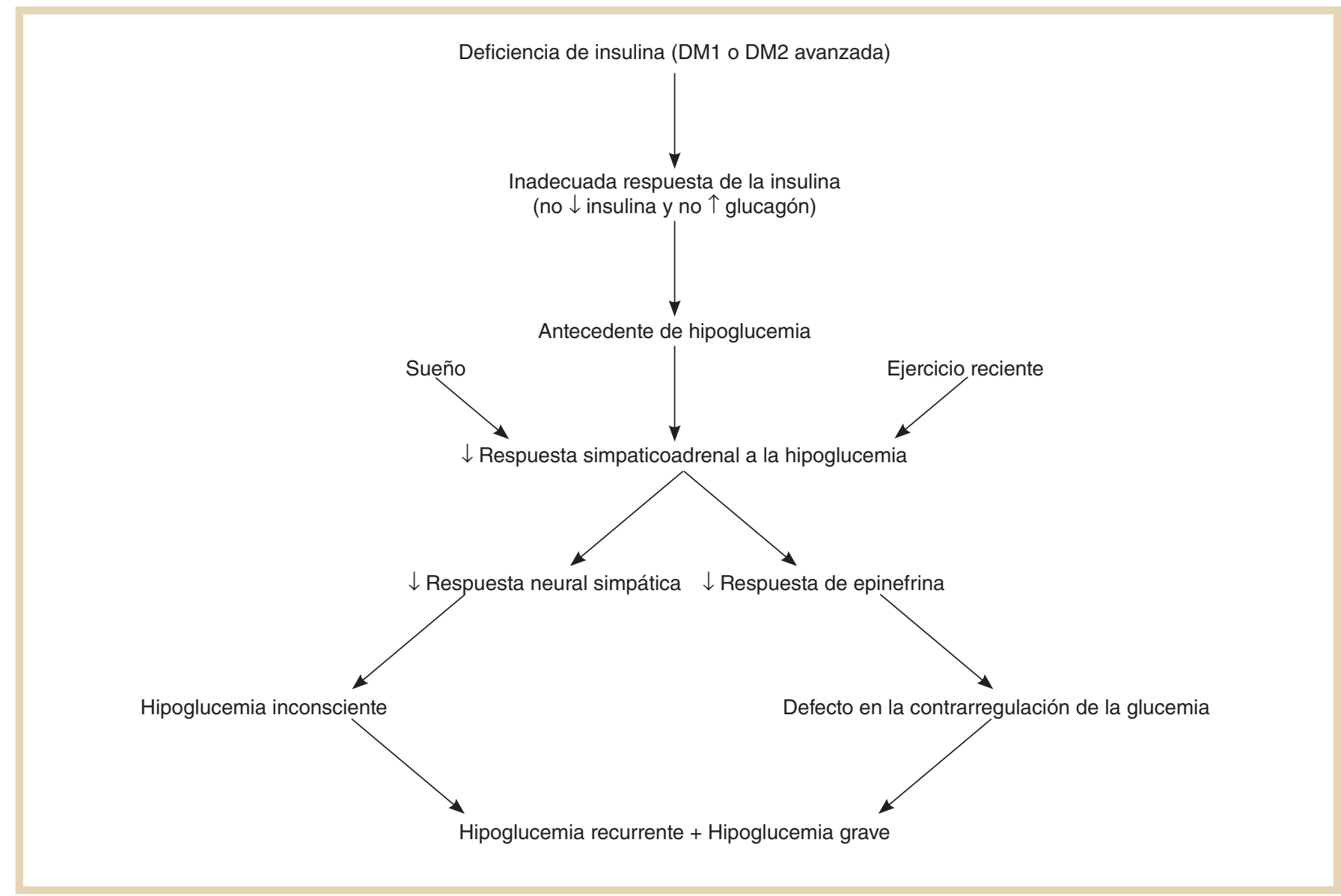

Figura 1. Vía de DAAH ladaptada de Finfer, et al., 201271). DAAH: disfunción autonómica asociada a hipoglucemia.

con insulina, sulfonilureas o glinidas ${ }^{58-61}$. La hipoglucemia iatrogénica resulta en morbilidad recurrente en gran parte de los pacientes con DM1 y en muchos con DM2 avanzada, puede ser letal ${ }^{62}$.

Los umbrales de respuesta a la hipoglucemia no solo varían entre individuos con DM, sino también en un mismo individuo, en función de la HbA1c y la experiencia de hipoglucemia. La ADA (Asociación Americana de la Diabetes) no define un valor numérico para el diagnóstico de la hipoglucemia, sino que la considera como «todos los episodios que se acompañen de una concentración plasmática anormalmente baja de glucosa que expone a un individuo a un daño potencial»63.
Concentraciones de glucosa entre 54 y $50 \mathrm{mg} / \mathrm{dl}$ inician el fenómeno de contrarregulación deficiente y de percepción de hipoglucemia, los componentes claves de la disfunción autonómica asociada a hipoglucemia (DAAH) en la diabetes ${ }^{64,65}$, que aumenta en 25 veces el riesgo de hipoglucemia grave y se puede asociar a episodios recientes de hipoglucemia, ejercicio previo o sueño que llevan a un déficit en la contrarregulación de la glucosa (principalmente a adrenalina) en cuanto a la respuesta autonómica y consecuentemente hipoglucemia inconsciente, lo que genera un ciclo vicioso de hipoglucemia recurrente ${ }^{65}$, como muestra la figura 1. Evitándose este nivel de glucemia puede revertirse la inconsciencia de la hipoglucemia y 
algunos otros aspectos de la contrarregulación deficiente ${ }^{66}$.

En la DM1, el no reconocer síntomas con el nivel de glucemia en $54 \mathrm{mg} / \mathrm{dl}$ incrementó cuatro veces el riesgo de hipoglucemia grave, definida como la necesidad de ayuda de un tercero para lograr la recuperacion $^{67}$. En la DM 1 y 2 estos niveles de glucemia se asocian a arritmias cardíacas ${ }^{68}$. En el estudio AC$\mathrm{CORD}^{7,69}$, la glucemia con valores de $50 \mathrm{mg} / \mathrm{dl}$ estaba asociada a mayor mortalidad, al igual que en el estudio ORIGIN ${ }^{70}$ y el NICESUGAR ${ }^{71}$.

Los signos y síntomas de hipoglucemia son clasificados como neurogénicos (predominantemente autonómicos) y neuroglucopénicos, con manifestaciones de cambio de conducta, convulsiones y coma. Generalmente, en condiciones normales, primero ocurren los síntomas neurogénicos y después los neuroglucopénicos. La percepción de la hipoglucemia ocurre por los síntomas neurogénicos: taquicardia, palidez, sudoración, hambre, parestesias, estremecimiento y ansiedad. La conciencia de esos síntomas es esencial para el tratamiento y prevenir la progresión a hipoglucemia grave. Los síntomas neuroglucopénicos más frecuentes son: disfunción cognitiva, cansancio, confusión mental, diplopía, alteraciones motoras (hemiparesia) y de comportamiento (violencia, disforia), hipotermia, convulsión y coma hasta muerte cerebral ${ }^{64}$.

Se resalta que la NAD debe ser diferenciada de la DAAH. Esta última es funcional y un fenómeno dinámico que puede ser revertido. La NAD es una alteración estructural e irreversible, con manifestaciones clínicas diversas ${ }^{1,65}$.

\section{TRATAMIENTO}

La prevención de la hipoglucemia es un desafío terapéutico en el tratamiento de la DM1 y en la DM2
TABla 8. Prevención de la hipoglucemia, conforme a las recomendaciones de la Asociación Americana de la Diabetes (ADA)-European Association for the Study of Diabetes (EASD)

1) Establecer metas glucémicas apropriadas 1.A HbA1c $<7 \%$

Jóvenes y pacientes recién diagnosticados sin comorbilidadades Independencia funcional

Sin lesiones (micro y/o macrovasculares)

1.B HbA1c $\sim 7.5$ a $8.0 \%$

Ancianos con más de 15 años de tiempo de diagnóstico

Historia de hipoglucemia grave

Comorbilidades graves (DRD con IRC) PNSD o NAC graves

2) Educar a los pacientes

Causas y cómo reconocer la hipoglucemia Autocuidado

Dieta y automonitorización proactiva

3) Sospecha de hipoglucemia

HbA1c < 5.5\%; HbA1c incoherente con antecedentes médicos Gastroparesia

Ganancia de peso con HbA1c baja

Actividad física nocturna excesiva

4) Utilizar agentes con bajo riesgo de hipoglucemia

DM2: MTF, TZD, iSGLT-2, aGLP-1, iDPP-4

DM1: Análogo de insulina basal, análogos de acción rápida o sistema de infusión continua de insulina

ADA: Asociación Americana de la Diabetes; AM: automonitoreo; aGLP-1: análogos del péptido similar al glucagón tipo 1; CH: carbohidratos; DM: diabetes mellitus; DRD: enfermedad renal diabética; EASD (Asociación Europea para los Estudio de la Diabetes); HbA1c: Prueba de hemoglobina glicosilada; iDPP-4: inhibidores de la dipeptidil peptidasa 4; IRC: enfermedad renal crónica; iSGLT-2: inhibidores del transportador sodio/glucosa; MTF: metformina; NAC: neuropatía autonómica cardiovascular; PNSD: polineuropatía simétrica distal.

Adaptada de Seaquist, et al., $2013^{63}$ y el International Hypoglycaemia Study Group, $2015^{67}$.

1. Automonitoreo de la glucemia capilar $>3$ veces al día

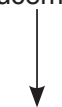

2. Tratar la hipoglucemia con dosis 3 veces mayores de ingesta de alimentos $(\mathrm{CH})(45 \mathrm{~g})$

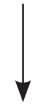

3. Objetivo inicial: evitar cualquier hipoglucemia durante 3 días

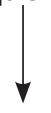

4. Objetivo posterior: evitar hipoglucemias durante 3 semanas

FIgURA 2. Abordaje secuencial de la DAAF ladaptada de Seaquist, et al., 2013 63 Yeoh, et al., 2015 ${ }^{66}$ y el International Hypoglycaemia Study Group, 201567). 
de larga evolución, y se torna crucial en los pacientes con DHAAH y NAD ${ }^{66,71,72}$. La tabla 8 contiene las recomendaciones actuales para prevenir la hipoglucemia en el tratamiento de la $\mathrm{DM}^{59,63,71}$.

\section{Automonitoreo de la glucemia capilar $>3$ veces al (4 a 7 veces). En el abordaje de la DAAH, el objetivo inicial es evitar glucemias $<100 \mathrm{mg} / \mathrm{dl}$ durante tres semanas, cuando debe ocurrir la recuperación de la respuesta simpaticoadrenérgica a la hipoglucemia (por lo menos parcialmente). En la figura 2 están resumidas las cuatro etapas, las llamadas normas secuenciales, para el abordaje de los pacientes con DAAH ${ }^{63,66,67}$.}

\section{BIBLIOGRAFÍA}

1. Spallone V, Ziegler D, Freeman R, Bernardi L, Frontoni S, Pop-Busui $\mathrm{R}$, et al.; Toronto Consensus Panel on Diabetic Neuropathy. Cardiovascular autonomic neuropathy in diabetes: clinical impact, assessment, diagnosis, and management. Diabetes Metab Res Rev. 2011;27: 639-53.

2. Escaño-Polanco FM, Odriozola A, Davidson J, Pedrosa H, Fuente G, Márquez G, et al. Consenso de Expertos para el Manejo de la Neuropatía Diabética. ALAD. 2016;6:121-50.

3. Pop-Busui R, Boulton AJM, Feldman EL, Bril V, Freeman R, Malik RA, et al. Diabetic Neuropathy: A position statement by the American Diabetes Association. Diabetes Care. 2017;40:136-54.

4. Maser RE, Mitchell BD, Vinik Al, Freeman R. The association between cardiovascular autonomic neuropathy and mortality in individuals with diabetes: a meta-analysis. Diabetes Care. 2003;26:1895-901.

5. Lykke JA, Tarnow L, Parving HH, Hilsted J. A combined abnormality in heart rate variation and QT corrected interval is a strong predictor of cardiovascular death in type 1 diabetes. Scand J Clin Lab Invest. 2008;68:654-9.

6. Pop-Busui R, Cleary PA, Braffett BH, Martin CL, Herman WH, Low PA, et al.; DCCT/EDIC Research Group. Association between cardiovascular autonomic neuropathy and left ventricular dysfunction: DCCT/ EDIC study (Diabetes Control and Complications Trial/Epidemiology of Diabetes Interventions and Complications). J Am Coll Cardiol. 2013; 61:447-54.

7. Pop-Busui R, Evans GW, Gerstein HC, Fonseca V, Fleg JL, Hoogwerf BJ, et al.; Action to Control Cardiovascular Risk in Diabetes Study Group. Effects of cardiac autonomic dysfunction on mortality risk in the Action to Control Cardiovascular Risk in Diabetes (ACCORD) trial. Diabetes Care. 2010;33:1578-84.

8. Young LH, Wackers FJ, Chyun DA, Davey JA, Barrett EJ, Taillefer R, et al.; DIAD Investigators. Cardiac outcomes after screening for asymptomatic coronary artery disease in patients with type 2 diabetes: the DIAD study: a randomized controlled trial. JAMA. 2009; 301:1547-55.

9. Ziegler D, Zentai CP, Perz S, Rathmann W, Haastert B, Döring A, et al.; KORA Study Group. Prediction of mortality using measures of cardiac autonomic dysfunction in the diabetic and nondiabetic population: the MONICA/ KORA Augsburg Cohort Study. Diabetes Care. 2008;31:556-61.

10. Jaiswal M, Divers J, Urbina EM, Dabelea D, Bell RA, ET SL. SEARCH for Diabetes in Youth Study Group. Cardiovascular autonomic neuropathy in adolescents and young adults with type 1 and type 2 diabetes: The SEARCH for Diabetes in Youth Cohort Study. Pediatr Diabetes. 2018 Jun;19(4):680-689. doi: 10.1111/pedi.12633. Epub 2018 Jan 2.

11. Ziegler D, Rathmann W, Dickhaus T, Meisinger C, Mielck A; KORA Study Group. Prevalence of polyneuropathy in pre-diabetes and diabetes is associated with abdominal obesity and macroangiopathy: the MONICA/KORA Augsburg Surveys S2 and S3. Diabetes Care. 2008;31: 464-9.

12. Carnethon MR, Prineas RJ, Temprosa M, Zhang ZM, Uwaifo G, Molitch $M E ;$ Diabetes Prevention Program Research Group. The association among autonomic nervous system function, incident diabetes, and intervention arm in the Diabetes Prevention Program. Diabetes Care. 2006;29:914-9.

13. Ziegler D, Voss A, Rathmann W, Strom A, Perz S, Roden M, et al.; KORA Study Group. Increased prevalence of cardiac autonomic dysfunction at different degrees of glucose intolerance in the general population: the KORA S4 survey. Diabetologia. 2015; 58:1118-28.

14. The effect of intensive diabetes therapy on measures of autonomic nervous system function in the Diabetes Control and Complications Trial (DCCT). Diabetologia. 1998;41:416-23.

15. Pop-Busui R, Low PA, Waberski BH, Martin CL, Albers JW, Feldman EL, et al.; DCCT/EDIC Research Group. Effects of prior intensive insulin therapy on cardiac autonomic nervous system function in type 1 diabetes mellitus: the Diabetes Control and Complications Trial/Epidemiology of Diabetes Interventions and Complications study (DCCT/ EDIC). Circulation. 2009;119:2886-93.

16. Ang L, Jaiswal M, Martin C, Pop-Busui R. Glucose control and diabetic neuropathy: lessons from recent large clinical trials. Curr Diab Rep. 2014;14:528.

17. Martin CL, Albers JW, Pop-Busui R; DCCT/ EDIC Research Group. Neuropathy and related findings in the Diabetes Control and Complications Trial/ Epidemiology of Diabetes Interventions and Complications study. Diabetes Care. 2014;37:31-8.

18. Low PA, Benrud-Larson LM, Sletten DM, Opfer-Gehrking TL, Weigand SD, O'Brien PC, et al. Autonomic symptoms and diabetic neuropathy: a population-based study. Diabetes Care. 2004;27:2942-7.

19. Marques JL, George E, Peacey SR, Harris ND, Macdonald IA, Cochrane $\mathrm{T}$, et al. Altered ventricular repolarization during hypoglycaemia in patients with diabetes. Diabet Med. 1997;14:648-54.

20. Robinson RT, Harris ND, Ireland RH, Macdonald IA, Heller SR. Changes in cardiac repolarization during clinical episodes of nocturnal hypoglycaemia in adults with type 1 diabetes. Diabetologia. 2004;47: 312-5.

21. Koivikko ML, Tulppo MP, Kiviniemi AM, Kallio MA, Perkiömäki JS, Salmela $\mathrm{PI}$, et al. Autonomic cardiac regulation during spontaneous nocturnal hypoglycemia in patients with type 1 diabetes. Diabetes Care. 2012;35:1585-90.

22. Astrup AS, Tarnow L, Rossing P, Hansen BV, Hilsted J, Parving HH. Cardiac autonomic neuropathy predicts cardiovascular morbidity and mortality in type 1 diabetic patients with diabetic nephropathy. Diabetes Care. 2006;29:334-9.

23. Orlov S, Cherney DZ, Pop-Busui R, Lovblom LE, Ficociello LH, Smiles AM, et al. Cardiac autonomic neuropathy and early progressive renal decline in patients with nonmacroalbuminuric type 1 diabetes. Clin J Am Soc Nephrol. 2015;10:1136-44.

24. Wheelock KM, Jaiswal M, Martin CL, Fufaa GD, Weil EJ, Lemley KV, et al. Cardiovascular autonomic neuropathy associates with nephropathy lesions in American Indians with type 2 diabetes. J Diabetes Complications. 2016;30:873-9. 
25. Neumann C, Schmid H. Relationship between the degree of cardiovascular autonomic dysfunction and symptoms of neuropathy and other complications of diabetes mellitus. Braz J Med Biol Res. 1995; 28(7):751-7.

26. Schmid H, Schaan B, Cecconello F, Maestri T, Neumann C. Proliferative diabetic retinopathy is related to cardiovascular autonomic neuropathy in non-insulin-dependent diabetes mellitus. Diabetes Res Clin Pract. 1995;29(3):163-8.

27. Neumann C, Martinez D, Schmid H. Nocturnal oxygen desaturation in diabetic patients with severe autonomic neuropathy. Diabetes Res Clin Pract. 1995;28(2):97-102.

28. Neumann C, Branchtein L, Schmid H. Severe autonomic neuropathy: how many symptoms? Diabetes Care. 1995;18(1):133-4.

29. Pop-Busui R. Cardiac autonomic neuropathy in diabetes: a clinical perspective. Diabetes Care. 2010;33:434-44.

30. Suarez GA, Opfer-Gehrking TL, Offord KP, Atkinson EJ, O'Brien PC, Low PA. The Autonomic Symptom Profile: a new instrument to assess autonomic symptoms. Neurology 1999;52:523-8.

31. Cryer PE. The barrier of hypoglycemia in diabetes. Diabetes. 2008; 57(12):3169-76.

32. Ewing DJ, Campbell IW, Clarke BF. Mortality in diabetic autonomic neuropathy. Lancet. 1976;1(7960):601-3.

33. Burgos LG, Ebert TJ, Asiddao C, Turner LA, Pattison CZ, Wang-Cheng $\mathrm{R}$, et al. Increased intraoperative cardiovascular morbidity in diabetics with autonomic neuropathy. Anesthesiology. 1989;70(4):591-7.

34. Veglio M, Sivieri R, Chinaglia A, Scaglione L, Cavallo-Perin P. QT interval prolongation and mortality in type 1 diabetic patients: a 5-year cohort prospective study. Neuropathy Study Group of the Italian Society of the Study of Diabetes, Piemonte Affiliate. Diabetes Care. 2000;23(9):1381-3.

35. Pop-Busui R, Kirkwood I, Schmid H, Marinescu V, Schroeder J, Larkin $D$, et al. Sympathetic dysfunction in type 1 diabetes: association with impaired myocardial blood flow reserve and diastolic dysfunction. J Am Coll Cardiol. 2004;44(12):2368-74.

36. Stevens MJ, Raffel DM, Allman KC, Dayanikli F, Ficaro E, Sandford T, et al. Cardiac sympathetic desinnervation in diabetes: implications for enhanced cardiovascular risk. Circulation. 1998;98(10):961-8.

37. Freeman R, Wieling W, Axelrod FB, Benditt DG, Benarroch E, Biaggioni I, et al. Consensus statement on the definition of orthostatic hypotension, neurally mediated syncope and the postural tachycardia syndrome. Auton Neurosci. 2011;161(1-2):46-8.

38. Gaspar L, Kruzliak P, Komornikova A, Celecova Z, Krahulec B, Balaz $D$, et al. Orthostatic hypotension in diabetic patients-10-year follow-up study. J Diabetes Complications. 2016;30(1):67-71.

39. Fleg JL, Evans GW, Margolis KL, Barzilay J, Basile JN, Bigger JT, et al. Orthostatic hypotension in the ACCORD (Action to Control Cardiovascular Risk in Diabetes) blood pressure trial: Prevalence, incidence, and prognostic significance. Hypertension. 2016;68(4):888-95.

40. Ewing DJ, Campbell IW, Clarke BF. Assessment of cardiovascular effects in diabetic autonomic neuropathy and prognostic implications. Ann Intern Med. 1980;92:308-11.

41. Consensus Committee of the American Autonomic Society and the American Academy of Neurology. Consensus statement on the definition of orthostatic hypotension, pure autonomic failure, and multiple system atrophy. Neurology. 1996;46:1470.

42. Witte DR, Tesfaye S, Chaturvedi N, Eaton SE, Kempler P, Fuller JH; EURODIAB Prospective Complications Study Group. Risk factors for cardiac autonomic neuropathy in type 1 diabetes mellitus. Diabetologia. 2005;48:164-70.

43. Rolim LCSP, Sá JR, Chacra AR, Dib AS. Diabetic cardiovascular autonomic neuropathy: risk factors, clinical impact and early diagnosis. Arq Bras Cardiol. 2008;90(4):e23-31.

44. Vinik Al, Ziegler D. Diabetic cardiovascular autonomic neuropathy. Circulation. 2007;115(3):387-97.
45. Burger AJ, Weinrauch LA, D`Elia JA, Aronson D. Effects of glycemic control on heart rate variability in type 1 diabetic patients with cardiac autonomic neuropathy. Am J Cardiol. 1999;84(6):687-91.

46. Gæde P, Oellgaard J, Carstensen B, Rossing P, Lund-Andersen H, Parving $\mathrm{HH}$, et al. Years of life gained by multifactorial intervention in patients with type 2 diabetes mellitus and microalbuminuria: 21 years follow-up on the Steno-2 randomised trial. Diabetologia. 2016; 59:2298-307.

47. Gaede P, Vedel P, Parving HH, Pedersen O. Intensified multifactorial intervention in patients with type 2 diabetes mellitus and microalbuminuria: the Steno type 2 randomised study. Lancet. 1999;353: 617-22.

48. Pagkalos M, Koutlianos N, Kouidi E, Pagkalos E, Mandroukas K, Deligiannis $A$. Heart rate variability modifications following exercise training in type 2 diabetic patients with definite cardiac autonomic neuropathy. Br J Sports Med. 2008;42:47-54.

49. Schaper NC, Andros G, Apelqvist J, Bakker K, Lammer J, Lepantalo M, et al.; International Working Group on Diabetic foot. Specific guidelines for the diagnosis and treatment of peripheral arterial disease in a patient with diabetes and ulceration of the foot 2011. Diabetes Metab Res ver. 2012;28(Suppl 1):236-7.

50. Choung RS, Locke GR 3rd, Schleck CD, Zinsmeister AR, Melton LJ 3rd, Talley NJ. Risk of gastroparesis in subjects with type 1 and 2 diabetes in the general population. Am J Gastroenterol. 2012;107:82-8.

51. Jones KL, Russo A, Stevens JE, Wishart JM, Berry MK, Horowitz M. Predictors of delayed gastric emptying in diabetes. Diabetes Care. 2001;24:1264-9.

52. Kempler P, Amarenco G, Freeman R. Management strategies for gastrointestinal, erectile, bladder, and sudomotor dysfunction in patients with diabetes. Diabetes Metab Res Rev. 2011;27:665-77.

53. Capon G. The impact of mellitus diabetes on the lower urinary tract: A review of Neuro-urology Committee of the French Association of Urology. Prog Urol. 2016;26(4):245-53.

54. Jackson RA, Vittinghoff E, Kanaya AM, Miles TP, Resnick HE, Kritchevsky SB, et al.; Health, Aging, and Body Composition Study. Urinary incontinence in elderly women: finding from the Health, Aging, and Body Composition Study. Obstet Gynecol. 2004;104: 301-7.

55. Michel MC, Mehlburger L, Schumacher H, Bressel HU, Goepel M. Effect of diabetes on lower urinary tract symptoms in patients with benign prostatic hyperplasia. J Urol. 2000;163:1725-9.

56. Van Den Eeden SK, Sarma AV, Rutledge BN, Cleary PA, Kusek JW, Nyberg LM, et al.; Diabetes Control and Complications Trial/Epidemiology of Diabetes Research Group. Effect of intensive glycemic control and diabetes complications on lower urinary tract symptoms in men with type 1 diabetes: DCCT/EDIC Interventions and Complications study. Diabetes Care. 2009;32:664-70.

57. Pop-Busui R, Hotaling J, Braffett BH, Cleary PA, Dunn RL, Martin CL, et al.; DCCT/EDIC Research Group. Cardiovascular autonomic neuropathy, erectile dysfunction and lower urinary tract symptoms in men with type 1 diabetes: findings from the DCCT/EDIC. J Urol. 2015;193: 2045-51.

58. Fedder J. Retrograde ejaculation and sexual dysfunction in men with diabetes mellitus: a prospective, controlled study. Andrology. 2013; 1(4):602-6.

59. Cryer PE. Glycemic goals in diabetes: trade-off between gly cemic control and iatrogenic hypoglycemia. Diabetes. 2014;63:218895.

60. Inzucchi SE, Bergenstal RM, Buse JB, Diamant M, Ferrannini E, Nauck $M$, et al. Management of hyperglycemia in type 2 diabetes, 2015: a patient-centered approach: update to a position statement of the American Diabetes Association and the European Association for the Study of Diabetes. Diabetes Care. 2015;38:140-9. 
61. Lipska KJ, Krumholz H, Soones T, Lee SJ. Polypharmacy in the aging patient: a review of glycemic control in older adults with type 2 diabetes. JAMA. 2016;315:1034-45.

62. Cryer P. Individualized glycemic goals and an expanded classification of severe hypoglycemia in diabetes. Diabetes Care. 2017;40:1641-3.

63. Seaquist ER, Anderson J, Childs B, Cryer P, Dagogo-Jack S, Fish L, et al. Hypoglycemia and diabetes: a report of a workgroup of the American Diabetes Association and The Endocrine Society. Diabetes Care. 2013;36:1384-95.

64. Cryer PE. Mechanisms of hypoglycemia associated autonomic failure in diabetes. N Engl J Med. 2013;369:362-72.

65. Cryer PE. Diverse causes of hypoglycemia-associated autonomic failure in diabetes. N Engl J Med. 2004;350(22):2272-9.

66. Yeoh E, Choudhary P, Nwokolo M, Ayis S, Amiel SA. Interventions that restore awareness of hypoglycemia in adults with type 1 diabetes: $A$ systematic review and meta-analysis. Diabetes Care. 2015;38(8):1592609.

67. International Hypoglycaemia Study Group. Minimizing hypoglycemia in diabetes. Diabetes Care. 2015;38(8):1583-91.
68. Chow E, Bernjak A, Williams S, Fawdry RA, Hibbert S, Freeman J, et al. Risk of cardiac arrhythmias during hypoglycemia in patients with type 2 diabetes and cardiovascular risk. Diabetes. 2014;63:1738-47.

69. Bonds DE, Miller ME, Bergenstal RM, Buse JB, Byington RP, Cutler JA, et al. The association between symptomatic, severe hypoglycaemia and mortality in type 2 diabetes: retrospective epidemiological analysis of the ACCORD study. BMJ. 2010;340:b4909.

70. ORIGIN Trial Investigators, Mellbin LG, Rydén L, Riddle MC, Probstfield J, Rosenstock J, Díaz R, et al. Does hypoglycaemia increase the risk of cardiovascular events? A report from the ORIGIN trial. Eur Heart J. 2013;34:3137-44.

71. NICE-SUGAR Study Investigators, Finfer S, Liu B, Chittock DR, Norton $\mathrm{R}$, Myburgh JA, McArthur C, et al. Hypoglycemia and risk of death in critically ill patients. N Engl J Med. 2012;367:1108-18.

72. International Hypoglycaemia Study Group. Glucose concentrations of less than $3.0 \mathrm{mmol} / \mathrm{l}(54 \mathrm{mg} / \mathrm{dl})$ should be reported in clinical trials: A joint position statement of the American Diabetes Association and the European Association for the Study of Diabetes. Diabetes Care. 2017;40:155-7. 